\title{
Comparative and Functional Analysis of Testis-Specific Genes
}

\author{
FuJun Liv, ShaoHua Jin, Ning Li, Xin Liu, HaiYan WANG, and JianYuan LI* \\ Shandong Research Centre for Stem Cell Engineering, Yu-Huang-Ding Hospital and Yan-Tai University; Yantai, Shandong \\ Province 264000, P. R. China. Received July 7, 2010; accepted September 29, 2010; published online October 13, 2010
}

The testis is the special male gonad responsible for spermatogenesis and steroidogenesis with complex gene expressions. Characterizing and comparing the testis-specific genes in different species can reveal key genes related to testis specifical functions and provide supplementary information for study of human testis function. We screened testis-specific genes from Unigene libraries, total 317, 449 and 147 testis-specific genes were identified for human, mouse and rat, respectively. Ten from thirteen selected human testis-specific genes were validated exclusively expressed in the testis by reverse transcription polymerase chain reaction (RT-PCR). Systematic bioinformatics analysis showed that specific genes were mainly related to spermatogenesis and testis development process with significant Glycolysis and Pyruvate metabolism. Enrichment functions were discussed.

Key words testis; specific; digital differential display

The testes produce the male gametes and the male sexual hormones which include all the processes involved in the production of gametes and the enzymatic reactions leading to the production of male steroid hormones. ${ }^{1)}$ These complicated processes of spermatogenesis and steroidogenesis reflected the complex gene expression in the testis. ${ }^{2,3}$ Many genes which were crucial for spermatogenesis and male fertility are often exclusively expressed in male germ cells. Some testis-specific genes have been screened including FAM46D, ${ }^{4}$ Adam $31,{ }^{5)}$ PDHA2, ${ }^{6}$ TEX101 and SPATA19, ${ }^{7)}$ etc. which have been proven to play critical roles in the process of spermatogenesis and steroidogenesis.

Various approaches have been used to analyze these tissueexclusively expressed genes including suppression subtractive hybridization $(\mathrm{SSH}){ }^{8)}$ differential display reverse transcription-polymerase chain reaction (RT-PCR), ${ }^{9)}$ microarray, ${ }^{10)}$ and whole genome approach analysis. ${ }^{11)}$ The UniGene database (http://www.ncbi.nlm.nih.gov/unigene) is a collection of sequences of individual clones from the cDNA libraries of various organs. The sequences are organized into clusters and each cluster is likely to contain sequence information of a single gene. Furthermore, the UniGene database includes information of various tissues, developmental, and diseases stages, and also provides valuable information about the putative tissue distribution of gene expression. Using the digital differential display (DDD) tool at the NCBI (http://www. ncbi.nlm.nih.gov/UniGene/ddd.cgi), some novel or specific genes had been identified. ${ }^{12,13)}$ Among various species, the highest number of expressed sequence tag (EST) sequences is available for the human and mouse, and most of knowledge of human testis physiology and function was obtained from research of homolog genes to mouse or rat. ${ }^{14)} \mathrm{Re}$ cently, we have mined the human, mouse and rat testis-specific gene expression patterns between testis and other organs in the UniGene library (www.ncbi.nlm.gov/unigene/info_ ddd.html) by digital differential display tool, and have made a comprehensive comparative and functional analysis of the results. This well-characterized database of testis-specific genes should serve as a source of reference for future research and clinical application.

\section{MATERIALS AND METHODS}

Digital Differential Display (DDD) Analysis of TestisSpecific Genes The DDD tool (http://www.ncbi.nlm.nih. gov/unigene/info_ddd.html) was performed to screen the testis-specific genes in human, mouse and rat between testis libraries and 18 other organ libraries. DDD tool takes advantage of UniGene by comparing the number of times that sequences from different libraries were assigned to a particular UniGene cluster. The testis and 18 other organs libraries used in the study were summarized and compared by DDD tool (Table 1). Subsequently, the genes with zero hits in the collected libraries (18 organs) from statistically significant difference results were designated as testis-specific genes.

Bioinformatics Analysis The DAVID tools 6.7 (http:// david.abcc.ncifcrf.gov/) was used to analyze the differential

Table 1. Summary of Human, Mouse and Rat Libraries Used in This Study

\begin{tabular}{lrrrrrr}
\hline \hline & \multicolumn{2}{c}{ Human } & \multicolumn{2}{l}{ Mouse } & \multicolumn{2}{l}{ Rat } \\
\cline { 2 - 7 } & Libraries & ESTs & Libraries & ESTs & Libraries & \multicolumn{1}{c}{ ESTs } \\
\hline Testis & 5 & 140944 & 7 & 67542 & 2 & 11732 \\
Other organs & & & & & & \\
$\quad$ Adipose & 2 & 9945 & 1 & 3135 & 1 & 4913 \\
Bladder & 1 & 8220 & 1 & 1597 & 0 & 0 \\
Bone & 1 & 6209 & 1 & 5439 & 0 & 0 \\
Brain & 11 & 179504 & 7 & 40381 & 6 & 20907 \\
Colon & 7 & 29584 & 1 & 5219 & 3 & 5458 \\
Heart & 4 & 19926 & 8 & 67542 & 4 & 31600 \\
Kidney & 6 & 49568 & 10 & 54164 & 4 & 24601 \\
Liver & 11 & 72628 & 6 & 25033 & 3 & 59093 \\
Lung & 7 & 46559 & 7 & 40355 & 2 & 8065 \\
Muscle & 2 & 6424 & 2 & 3031 & 1 & 2429 \\
Ovary & 4 & 10862 & 2 & 6847 & 1 & 4443 \\
Pancreas & 1 & 4308 & 6 & 22020 & 1 & 16173 \\
Placenta & 12 & 71806 & 7 & 21182 & 2 & 8475 \\
Prostate & 5 & 63083 & 0 & 0 & 1 & 9076 \\
Spleen & 2 & 36689 & 3 & 46301 & 1 & 3629 \\
Stomach & 11 & 36759 & 4 & 18360 & 0 & 0 \\
Thymus & 4 & 72916 & 8 & 93976 & 0 & 0 \\
$\quad$ Uterus & 5 & 58541 & 0 & 0 & 0 & 0 \\
Total & 96 & 783531 & 74 & 454582 & 30 & 198862 \\
\hline
\end{tabular}

Libraries from 18 organs against testis libraries were used in the digital differential display (DDD) program. 
Table 2. Lists of Primers Used in RT-PCR to Confirm Gene Transcripts of Testis-Specific Genes

\begin{tabular}{|c|c|c|c|}
\hline Unigene & Forward primers $\left(5^{\prime}-3^{\prime}\right)$ & Reverse primers $\left(5^{\prime}-3^{\prime}\right)$ & Production $(\mathrm{bp})$ \\
\hline Hs. 126780 & TCAACGAGCAATACGAGCAG & GGAGGTGGGAGCGGATAA & 489 \\
\hline Hs.472491 & CCGTCGCATCGTCTGTTC & GCCTTCCACCCTTTCACC & 321 \\
\hline Hs. 490715 & CCCGAGTGATACACGATG & AGGATGCGGTGGCTGATT & 187 \\
\hline Hs. 632402 & ССТТТССТАТСТСССТСА & СTCTTGCTCCAATCTCCT & 200 \\
\hline Hs. 149030 & TTTGCCCGAACCCGATTA & GCCGTTTAGCCTCACCAC & 337 \\
\hline Hs.97542 & AGATTGACTGCTGGGATG & GTGCCTCTGGTGATGGTA & 270 \\
\hline Hs.41101 & GACATTTGCCATTCTATC & AGTTACCGTCCAAGTTTT & 190 \\
\hline Hs. 126838 & CTTAGGTGACCGTGTTGG & ATGGCAGTGATGTTTATTGT & 352 \\
\hline Hs. 177959 & CATTTGGCAAAGAAGTAG & AACCCAAGTATGAAGAAC & 345 \\
\hline Hs.525769 & CTGCCTGGTGGCTGATAG & TGGGCTGAGACAGACATCG & 334 \\
\hline Hs.497034 & CTGGTCCTATCGCACAAC & AAGCCAAATCCAAATCAC & 354 \\
\hline \multicolumn{4}{|l|}{ Mm.436562, Rn.64496 homolog } \\
\hline to human Gapdhs & ACCCACTCGTCCATCTTCG & TCCCGTTTCACTTGTCTCG & 151 \\
\hline \multicolumn{4}{|l|}{ Mm.143889, Rn.92321 homolog } \\
\hline to human $A D A M 5$ & ATTACCATAGGGAAAGCC & TGAGTCCTGAACATACGC & 197 \\
\hline$\beta$-Actin & GCTGTCACCTTCACCGTTCC & GCTGTCACCTTCACCGTTCC & 268 \\
\hline
\end{tabular}

Thirteen genes were selected randomly to confirm its expressions in different human organs.

functional annotations including molecular functions, Biological processes, cellular component, KEGG pathway and functional Clustering. Background was Homo sapiens, Mus musculus and Rattus norvegicus, Fisher Exact test is used to determine significant results.

RT-PCR Analysis Eleven human genes (Hs.126780, Hs.472491, Hs.490715, Hs.632402, Hs.149030, Hs.97542, Hs.41101, Hs.126838, Hs.177959, Hs.525769, Hs.497034) and two homolog genes (Mm.436562 and Rn.64496 Mm homolog to human gene Gapdhs, 143889 and Rn.92321 homolog to human gene $A D A M 5$ ) were selected for testis-specific validation (Table 2). Forward and reverse primers for the genes were designed using primer premier 5.0 Software (PREMIER Biosoft International) on the basis of sequences published in GenBank. Gene expression was examined by RT-PCR in the 12 organs (testis, heart, liver, spleen, lung, kidney, stomach, muscle, adipose, placenta, ovary and colon) from accident victims (27-32 years old) who had died in car accidents and were collected while artificial circulation maintained organs assigned for transplantation. ${ }^{15)}$ All procedures were approved by the Ethics Committee of Yu-HuangDing Hospital.

\section{RESULTS}

Characteristic of Testis-Specific Genes Comparison of expressed sequence tags (ESTs) between testis and 18 other organs was showed in Table 1. 140944 human testis ESTs (5 libraries) and 783531 ESTs from other organs (95 libraries) were used to screen 317 human testis-specific genes including 38 unknown genes. 67542 mouse testis ESTs (7 libraries) and 454582 other organs ESTs (74 libraries) were used to screen 449 mouse testis-specific genes including 45 unknown genes. 11732 rat testis ESTs (2 libraries) and 198862 other organ ESTs (30 libraries) were used to screen 147 rat testisspecific genes including 8 unknown genes (Table 3 ).

Twenty specific genes were co-expressed in human, mouse and rat testis, which included five genes common in the human sperm proteome ${ }^{16)}$ (Table 4, Fig. 1). Comparison of human testis-specific genes with human sperm proteome showed 33 specific genes overlapped in the two database
(Table 5).

Functional Analysis The testis-specific genes (Unigene IDs) were imported into DAVID Function Annotation lists. Total 282, 442 and 140 Unigene IDs were converted into DAVID IDs, respectively of the human, mouse and rat specific genes. Common statistically significant functions including molecular functions, biological processes, KEGG pathway and functional clustering were displayed in Fig. 2. Results showed that specific genes were mainly related to spermatogenesis and development process with significant Glycolysis and Pyruvate metabolism.

RT-PCR Analysis Analysis of selected thirteen genes by RT-PCR confirmed that ten genes including two homolog genes were specifically expressed in the testis tissues. Hs.490715 was also weakly expressed in ovary, Hs.632402 was also detected in muscle, and Hs.149030 was expressed in liver and spleen (Fig. 3).

\section{DISCUSSION}

In the present study, eighteen organs libraries were pooled to compare with testis libraries of human, mouse and rat, respectively. The designation of testis-specific genes was only given if the gene had zero hits in the collected libraries (18 organs) from statistically significant difference results generated by DDD tool. The design guaranteed the significance of the results as much as possible. However, the results showed low overlap among human, mouse and rat testis-specific genes, this phenomenon maybe partially due to the number disparities between libraries and partially due to differential gene expressions among species or alternative splicing which included $30 \%$ in human testis ESTs. ${ }^{17)}$ Comparative analysis of human testis-specific genes in this study with Tang's report ${ }^{18)}$ only 76 overlapped genes were found. Low overlap maybe mainly due to database update which generally takes less than one month and more rigorous screening methods we used. Comparison of 139 (47\%) unknown genes in TANG' report and 38 (11\%) unknown genes in present study indicated the current database were annotated more detailed. Baker reported 976 from 1053 proteins detected in spermatozoa demonstrated EST expressions in the testis. Conversely, 
Table 3. Lists of Unigene IDs of Human, Mouse and Rat Testis-Specific Genes

Unigene IDs of 317 human testis-specific genes

\begin{tabular}{|c|c|c|c|c|c|c|c|c|}
\hline Hs. 107944 & Hs. 131098 & Hs. 213307 & Hs.367764 & Hs. 456833 & Hs. 558087 & Hs. 643584 & Hs.666581 & Hs.98394 \\
\hline Hs. 111850 & Hs.131179 & Hs. 220558 & Hs.367959 & Hs.459153 & Hs. 558560 & Hs. 644625 & Hs.671096 & Hs.98586 \\
\hline Hs. 112645 & Hs.131361 & Hs. 223581 & Hs.369473 & Hs. 459534 & Hs. 558748 & Hs. 645245 & Hs.675821 & Hs.98712 \\
\hline Hs. 112651 & Hs.131469 & Hs. 226805 & Hs.369765 & Hs.472491 & Hs.567414 & Hs. 645542 & Hs.67639 & Hs.98848 \\
\hline Hs. 112680 & Hs. 131615 & Hs. 236635 & Hs. 371738 & Hs. 475243 & Hs.567642 & Hs. 646421 & Hs.680123 & Hs.99161 \\
\hline Hs. 112743 & Hs.131654 & Hs. 240053 & Hs.375039 & Hs.481369 & Hs.567674 & Hs. 646533 & Hs.689473 & \\
\hline Hs. 113290 & Hs.131824 & Hs. 245476 & Hs.376008 & Hs. 482520 & Hs.567774 & Hs. 646574 & Hs.689547 & \\
\hline Hs. 113291 & Hs.133095 & Hs. 247868 & Hs.376505 & Hs.487409 & Hs.568279 & Hs. 647077 & Hs.694285 & \\
\hline Hs. 115429 & Hs. 136555 & Hs. 2633 & Hs.378893 & Hs.490715 & Hs.568772 & Hs. 648235 & Hs.701555 & \\
\hline Hs. 115460 & Hs.13861 & Hs. 274285 & Hs.381087 & Hs.491734 & Hs.568804 & Hs. 648270 & Hs.707861 & \\
\hline Hs. 118205 & Hs. 142003 & Hs. 283011 & Hs.388827 & Hs.491885 & Hs.571446 & Hs. 648337 & Hs. 708818 & \\
\hline Hs. 119207 & Hs.143519 & Hs. 283096 & Hs.388841 & Hs.494981 & Hs. 572187 & Hs. 652956 & Hs.709890 & \\
\hline Hs. 120316 & Hs. 145601 & Hs. 283764 & Hs.388874 & Hs.497034 & Hs. 572495 & Hs. 654377 & Hs. 711267 & \\
\hline Hs. 120319 & Hs. 146544 & Hs. 298312 & Hs.390221 & Hs. 510812 & Hs.575609 & Hs. 655170 & Hs.712915 & \\
\hline Hs. 120364 & Hs.146614 & Hs.302028 & Hs.398196 & Hs.511849 & Hs.584289 & Hs. 657342 & Hs.714939 & \\
\hline Hs. 120391 & Hs. 147276 & Hs.303923 & Hs.405628 & Hs. 511983 & Hs. 585048 & Hs. 658505 & Hs.716685 & \\
\hline Hs. 120831 & Hs.147816 & Hs.304020 & Hs.405659 & Hs. 514820 & Hs.585464 & Hs. 658630 & Hs.719419 & \\
\hline Hs. 121021 & Hs. 148250 & Hs.307052 & Hs.406696 & Hs. 517091 & Hs.587755 & Hs.659889 & Hs. 720893 & \\
\hline Hs. 121084 & Hs. 149030 & Hs.307086 & Hs.408336 & Hs.518957 & Hs.591011 & Hs. 660607 & Hs. 721200 & \\
\hline Hs. 121200 & Hs. 151167 & Hs.307999 & Hs.41101 & Hs.519507 & Hs.591296 & Hs. 660774 & Hs. 721231 & \\
\hline Hs. 121438 & Hs. 155942 & Hs.320892 & Hs.411239 & Hs.519941 & Hs.591738 & Hs.661266 & Hs.721828 & \\
\hline Hs. 121494 & Hs. 159043 & Hs. 324335 & Hs.412818 & Hs. 520554 & Hs.591941 & Hs.662013 & Hs.721999 & \\
\hline Hs. 122511 & Hs.160594 & Hs. 326528 & Hs.414648 & Hs. 522093 & Hs. 592008 & Hs.662199 & Hs.722049 & \\
\hline Hs. 122986 & Hs.169222 & Hs.333132 & Hs.424594 & Hs. 524331 & Hs. 592128 & Hs. 662205 & Hs. 722203 & \\
\hline Hs. 123530 & Hs.169797 & Hs. 335788 & Hs.431161 & Hs.524406 & Hs. 620508 & Hs. 662212 & Hs.722222 & \\
\hline Hs. 123536 & Hs.177959 & Hs.343487 & Hs.433336 & Hs. 525666 & Hs.629246 & Hs. 662213 & Hs. 722843 & \\
\hline Hs. 125251 & Hs. 179646 & Hs.343631 & Hs.434715 & Hs.525769 & Hs.629498 & Hs. 662219 & Hs. 723454 & \\
\hline Hs. 126035 & Hs. 186363 & Hs. 348263 & Hs.435371 & Hs. 528454 & Hs.630533 & Hs. 662288 & Hs.79363 & \\
\hline Hs. 126730 & Hs. 189183 & Hs. 348618 & Hs.437006 & Hs. 528847 & Hs.631533 & Hs.662304 & Hs. 82921 & \\
\hline Hs. 126780 & Hs. 189184 & Hs. 348700 & Hs.437141 & Hs. 529965 & Hs.631621 & Hs. 662373 & Hs.97335 & \\
\hline Hs. 126838 & Hs.190267 & Hs. 349758 & Hs.439998 & Hs. 530358 & Hs.631667 & Hs. 662737 & Hs.97408 & \\
\hline Hs. 127882 & Hs. 192090 & Hs. 350507 & Hs. 444230 & Hs. 534390 & Hs.631931 & Hs. 662766 & Hs.97501 & \\
\hline Hs. 128069 & Hs.194610 & Hs. 350671 & Hs.444236 & Hs. 535004 & Hs. 632070 & Hs. 663128 & Hs. 97542 & \\
\hline Hs. 128574 & Hs. 197354 & Hs. 351068 & Hs.447948 & Hs. 535216 & Hs.632233 & Hs.664011 & Hs. 97633 & \\
\hline Hs. 129293 & Hs.197801 & Hs. 351815 & Hs.448583 & Hs. 535454 & Hs.632402 & Hs.664098 & Hs. 97714 & \\
\hline Hs. 129435 & Hs.2042 & Hs. 357567 & Hs.448884 & Hs. 535667 & Hs. 632574 & Hs.664194 & Hs.97726 & \\
\hline Hs. 130672 & Hs.209206 & Hs.363054 & Hs.453629 & Hs. 535746 & Hs.632635 & Hs. 665663 & Hs.97933 & \\
\hline Hs. 130722 & Hs.209542 & Hs. 367245 & Hs.454647 & Hs. 537348 & Hs.637057 & Hs.666095 & Hs. 98008 & \\
\hline Hs. 130949 & Hs. 210377 & Hs.367727 & Hs.454835 & Hs.539964 & Hs.641085 & Hs.666099 & Hs.98025 & \\
\hline
\end{tabular}

Unigene IDs of 449 mouse testis-specific genes

\begin{tabular}{|c|c|c|c|c|c|c|c|c|}
\hline Mm.189660 & Mm.189390 & Mm.251434 & Mm.287421 & Mm.33648 & Mm.422869 & Mm.45373 & Mm.478333 & Mm.7411 \\
\hline Mm.10725 & Mm.189648 & Mm.251601 & Mm.287783 & Mm.33683 & $\mathrm{Mm} .423014$ & Mm.45377 & Mm.478493 & Mm.74465 \\
\hline Mm.107718 & Mm.189668 & Mm.252110 & Mm.291521 & Mm.338103 & Mm.425024 & Mm.45442 & Mm.4815 & Mm.75060 \\
\hline Mm.107918 & Mm.189696 & Mm.252733 & Mm.291723 & Mm.33811 & Mm.425335 & Mm.45462 & Mm.4872 & Mm.75066 \\
\hline Mm.108054 & Mm.195732 & Mm.252738 & Mm.291732 & Mm.33864 & Mm.425687 & Mm.45611 & Mm.50087 & Mm.76826 \\
\hline Mm.108632 & Mm.195831 & Mm.252761 & Mm.291887 & Mm.339192 & Mm.425989 & Mm.45615 & Mm.50108 & Mm.76859 \\
\hline Mm.108729 & Mm.196288 & Mm.252823 & Mm.292326 & Mm.34166 & Mm.425994 & Mm.45820 & Mm.50361 & Mm.78341 \\
\hline Mm.116705 & Mm.196313 & Mm.255732 & Mm.293018 & Mm.341880 & Mm.42733 & Mm.45824 & Mm.50808 & Mm.78368 \\
\hline Mm.116803 & Mm.196315 & Mm.261400 & Mm.293026 & Mm.347413 & Mm.431297 & Mm.45833 & Mm.5168 & Mm.78551 \\
\hline Mm.116871 & Mm.204664 & Mm.26189 & Mm.293080 & Mm.348215 & Mm.432087 & Mm.458917 & Mm.52511 & Mm.78900 \\
\hline Mm.116957 & Mm.206798 & Mm.263615 & Mm.29625 & Mm.34841 & Mm.432764 & Mm.460742 & Mm.52550 & Mm.78965 \\
\hline Mm.120271 & Mm.21005 & Mm.263642 & Mm.296649 & Mm.349600 & Mm.436562 & Mm.46102 & Mm.55591 & Mm.79031 \\
\hline Mm.121570 & Mm.212777 & Mm.263706 & Mm.29788 & Mm.35644 & Mm.436668 & Mm.46106 & Mm.55734 & Mm.79070 \\
\hline Mm.12743 & Mm.213909 & Mm.263708 & Mm.297977 & Mm.358754 & Mm.436785 & Mm.46114 & Mm.55827 & Mm.79072 \\
\hline Mm.128134 & Mm.214178 & Mm.264645 & Mm.30482 & Mm.360540 & Mm.437189 & Mm.46117 & Mm.55848 & Mm.79114 \\
\hline Mm.12817 & Mm.214599 & Mm.264651 & Mm.30494 & Mm.371643 & Mm.439708 & Mm.46124 & Mm.55870 & Mm.79179 \\
\hline Mm.1296 & Mm.217475 & Mm.266280 & Mm.307436 & Mm.373292 & Mm.439725 & Mm.46125 & Mm.55891 & Mm.80075 \\
\hline Mm.140115 & Mm.229259 & Mm.2688 & Mm.308822 & Mm.373573 & Mm.439815 & Mm.46129 & Mm.55982 & Mm.8019 \\
\hline Mm.1404 & Mm.23166 & Mm.268996 & Mm.30958 & Mm.379188 & Mm.439819 & Mm.46132 & Mm.56404 & Mm.8122 \\
\hline Mm.141235 & Mm.232593 & Mm.269223 & Mm.30959 & Mm.37953 & Mm.439983 & Mm.46136 & Mm.56430 & Mm.8149 \\
\hline Mm.141275 & Mm.23361 & Mm.26987 & Mm.310201 & Mm.380214 & Mm.440041 & Mm.46140 & Mm.56514 & Mm. 82775 \\
\hline Mm.141324 & Mm.23377 & Mm.26988 & Mm.314688 & Mm.380836 & Mm.440232 & Mm.46144 & Mm.60362 & Mm. 82820 \\
\hline Mm.141383 & Mm.23385 & Mm.27023 & Mm.31700 & Mm.382859 & Mm.440412 & Mm.46158 & Mm.60688 & Mm.84331 \\
\hline Mm.141451 & Mm.23397 & Mm.27027 & Mm.31708 & Mm.383221 & Mm.440422 & Mm.46159 & Mm.60690 & Mm.84467 \\
\hline Mm.141471 & Mm.23401 & Mm.270295 & Mm.318133 & Mm.383770 & Mm.440432 & Mm.46166 & Mm.60992 & Mm.84502 \\
\hline Mm.142735 & Mm.235024 & Mm.271255 & Mm.320172 & Mm.384235 & Mm.440453 & Mm.46167 & Mm.61131 & Mm.84522 \\
\hline Mm.143764 & Mm.23509 & Mm.271285 & Mm.320289 & Mm.386790 & Mm.440554 & Mm.46170 & Mm.61148 & Mm.84567 \\
\hline
\end{tabular}


Table 3. Continued

Unigene IDs of 317 human testis-specific genes

\begin{tabular}{|c|c|c|c|c|c|c|c|c|}
\hline Mm.143802 & Mm.23510 & Mm.272119 & Mm.32231 & Mm.386795 & Mm.440760 & Mm.46175 & Mm.61206 & Mm. 84725 \\
\hline Mm.143889 & Mm.23515 & Mm.272226 & Mm.32242 & Mm.386810 & Mm.441372 & Mm.46405 & Mm.61243 & Mm.84774 \\
\hline Mm.1453 & Mm.23516 & Mm.272519 & Mm.325325 & Mm.386907 & Mm.441493 & Mm.46470 & Mm.61330 & Mm. 85045 \\
\hline Mm.148848 & Mm.23520 & Mm. 272720 & Mm.325769 & Mm.387525 & Mm.441494 & Mm.467143 & Mm.6211 & Mm.8637 \\
\hline Mm.1498 & Mm.23522 & Mm. 272723 & Mm.329132 & Mm.389085 & Mm. 441500 & Mm.467144 & Mm. 65550 & Mm.87067 \\
\hline Mm.154333 & Mm.23534 & Mm.272746 & Mm.329435 & Mm.389164 & Mm.441963 & Mm.467152 & Mm.660 & Mm.87320 \\
\hline Mm.157323 & Mm.23547 & Mm.272766 & Mm.330943 & Mm.389509 & Mm.442295 & Mm.467157 & Mm.66794 & Mm.87321 \\
\hline Mm.158148 & Mm.235535 & Mm.272794 & Mm.331893 & Mm.389676 & Mm.443238 & Mm.467163 & Mm.66894 & Mm.87325 \\
\hline Mm.158494 & Mm.235716 & Mm.272843 & Mm.332291 & Mm.389950 & Mm.443268 & Mm.467176 & Mm.66952 & Mm.87331 \\
\hline Mm.159193 & Mm.23887 & Mm.27287 & Mm.332570 & Mm.390691 & Mm.443270 & Mm.467190 & Mm.67234 & Mm.87352 \\
\hline Mm.159404 & Mm.239376 & $\mathrm{Mm} .272871$ & Mm.332809 & Mm.390750 & Mm.443272 & Mm.467193 & Mm.67300 & Mm.87357 \\
\hline Mm.159949 & Mm.243849 & Mm.273301 & Mm.333010 & Mm.390785 & Mm.443285 & Mm.467210 & Mm.67595 & Mm.87365 \\
\hline Mm.160088 & Mm.24454 & Mm.273316 & Mm.333542 & Mm.391200 & Mm.443287 & Mm.4688 & Mm.67628 & Mm. 87382 \\
\hline Mm.160106 & Mm.246123 & Mm.273327 & Mm.334092 & Mm.391427 & Mm.443291 & Mm.473053 & Mm.67665 & Mm.87419 \\
\hline Mm.160714 & Mm.248902 & Mm.275677 & Mm.334107 & Mm.391486 & Mm.443311 & Mm.473700 & Mm.67677 & Mm.87446 \\
\hline Mm.16563 & Mm.249912 & $\mathrm{Mm} .276086$ & Mm.334425 & Mm.391543 & Mm.444289 & Mm.475127 & Mm.69388 & Mm.87448 \\
\hline Mm.173395 & Mm.250430 & Mm.276332 & Mm.334503 & Mm.392402 & Mm.444291 & Mm.475210 & Mm.717 & Mm.87624 \\
\hline Mm.17413 & Mm.250432 & Mm.276558 & Mm.335431 & Mm.393774 & Mm.444650 & Mm.475302 & Mm.71906 & Mm. 87748 \\
\hline Mm.17621 & Mm.250447 & Mm.276780 & Mm.335525 & Mm.394644 & Mm.444671 & Mm.476887 & Mm.72491 & Mm.89197 \\
\hline Mm.17649 & Mm.250465 & Mm.279725 & Mm.335855 & Mm.398302 & Mm.444704 & Mm.477010 & Mm.72959 & Mm.89943 \\
\hline Mm.178707 & Mm.250837 & Mm.279939 & Mm.33629 & Mm.415388 & Mm.44896 & Mm.477086 & Mm.72970 & Mm.9493 \\
\hline Mm.179050 & Mm.251303 & Mm.280878 & Mm.33631 & Mm.4223 & Mm.45302 & Mm.477599 & Mm.73205 & Mm.9500 \\
\hline Mm.179144 & Mm.251409 & Mm.282330 & Mm.33646 & Mm.422673 & Mm.45306 & Mm.4776 & Mm.73222 & \\
\hline
\end{tabular}

Unigene IDs of 147 rat testis-specific genes

\begin{tabular}{|c|c|c|c|c|c|c|c|c|}
\hline Rn.10423 & Rn.122594 & Rn.126314 & Rn.155649 & Rn.18131 & Rn.208235 & Rn.27474 & Rn.50288 & Rn.66649 \\
\hline Rn.105169 & Rn.122663 & Rn.127514 & Rn.159914 & Rn. 187184 & Rn.208286 & Rn.27738 & Rn.50442 & Rn.67823 \\
\hline Rn.107052 & Rn.122669 & Rn.136809 & Rn.159999 & Rn.195918 & Rn.208808 & Rn. 27773 & Rn.50944 & Rn.68598 \\
\hline Rn.108313 & Rn.122802 & Rn.136989 & Rn.160028 & Rn.198212 & Rn.209597 & Rn.3253 & Rn.51329 & Rn.81074 \\
\hline Rn.108510 & Rn.122806 & Rn.137100 & Rn.160149 & Rn.198417 & Rn.209823 & Rn.33200 & Rn.51791 & Rn.83796 \\
\hline Rn.108545 & Rn.123031 & Rn.137247 & Rn.160929 & Rn.198431 & Rn.210834 & Rn.34654 & Rn.51883 & Rn.83901 \\
\hline Rn.108612 & Rn.123117 & Rn.137540 & Rn.160960 & Rn.198484 & Rn.210888 & Rn.38091 & Rn.53634 & Rn.8467 \\
\hline Rn.109395 & Rn.123156 & Rn.137580 & Rn.161900 & Rn.198886 & Rn.212099 & Rn.42917 & Rn.54001 & Rn.92321 \\
\hline Rn.109532 & Rn.123187 & Rn.138402 & Rn.162390 & Rn.198897 & Rn.214118 & Rn.42918 & Rn.55448 & Rn.92436 \\
\hline Rn.110654 & Rn.123202 & Rn.138828 & Rn. 162573 & Rn.199021 & Rn.215004 & Rn.43509 & Rn.57137 & Rn.95004 \\
\hline Rn.11126 & Rn. 123222 & Rn.144145 & Rn.162717 & Rn.201753 & Rn.215007 & Rn. 45038 & Rn.57304 & Rn.96432 \\
\hline Rn.111442 & Rn. 123227 & Rn.145908 & Rn.162868 & Rn.202679 & Rn.215015 & Rn.45119 & Rn.58091 & \\
\hline Rn.111731 & Rn.123299 & Rn.146844 & Rn.163190 & Rn.202733 & Rn.215017 & Rn.46772 & Rn.59403 & \\
\hline Rn.113616 & Rn.123316 & Rn.150800 & Rn.163425 & Rn.205065 & Rn.215025 & Rn.47152 & Rn.60723 & \\
\hline Rn.113868 & Rn.123383 & Rn.154599 & Rn.170874 & Rn.205660 & Rn.217373 & Rn.47921 & Rn.63585 & \\
\hline Rn.114052 & Rn. 123420 & Rn.155129 & Rn.172256 & Rn.206234 & Rn.219514 & Rn.48706 & Rn. 6427 & \\
\hline Rn.119400 & Rn.125706 & Rn.155462 & Rn.172733 & Rn.20672 & Rn.26693 & Rn.50006 & Rn.64496 & \\
\hline
\end{tabular}

Total of 317,449 and 147 Unigenes were identified through the program mentioned above.

Table 4. Common Testis-Specific Genes in Human, Mouse and Rat Testis

\begin{tabular}{|c|c|c|c|}
\hline Unigene & Gene name & Gene description & Species \\
\hline Hs.98008; Mm.61206; Rn.108510 & *GK2 & Glycerol kinase 2 & Human, mouse, rat \\
\hline Hs.115460; Mm.348215; Rn.109532 & CCIN & Calicin & Human, mouse, rat \\
\hline Hs. $131361 ;$ Mm.4223; Rn. 11126 & PDHA2 & Pyruvate dehydrogenase alpha 2 & Human, mouse, rat \\
\hline Hs.226805; Mm.67677; Rn.113616 & KIF2B & Kinesin family member $2 \mathrm{~B}$ & Human, mouse, rat \\
\hline Hs.236635; Mm.30958; Rn.119400 & *ACTRT2 & Actin-related protein $\mathrm{T} 2$ & Human, mouse, rat \\
\hline Hs.131098; Mm.87624; Rn.123117 & FAM71F1 & Family with sequence similarity 71 , member F1 & Human, mouse, rat \\
\hline Hs.335788; Mm.307436; Rn. 123420 & ALS2CR11 & Amyotrophic lateral sclerosis 2 chromosome region, candidate 11 & Human, mouse, rat \\
\hline Hs.186363; Mm.23361; Rn.136809 & SPERT & Spermatid associated & Human, mouse, rat \\
\hline Hs.120319; Mm.272843; Rn. 137540 & NT5C1B & $5^{\prime}$-Nucleotidase, cytosolic IB & Human, mouse, rat \\
\hline Hs.41101; Mm.296649; Rn.150800 & CCDC110 & Coiled-coil domain containing 110 & Human, mouse, rat \\
\hline Hs.518957; Mm.8122; Rn.162573 & ADAD1 & Adenosine deaminase domain containing 1 & Human, mouse, rat \\
\hline Hs.307052; Mm.475210; Rn.208286 & *LDHAL6B & Lactate dehydrogenase A-like 6B & Human, mouse, rat \\
\hline Hs.487409; Mm.441372; Rn.210888 & PAPOLB & Poly polymerase beta & Human, mouse, rat \\
\hline Hs.647077; Mm.141471; Rn.215017 & GALNTL5 & $\begin{array}{l}\text { UDP- } N \text {-acetyl-alpha-D-galactosamine : polypeptide } \\
N \text {-acetylgalactosaminyltransferase-like } 5\end{array}$ & Human, mouse, rat \\
\hline Hs.437141; Mm.386907; Rn.50442 & CCDC63 & Coiled-coil domain containing 63 & Human, mouse, rat \\
\hline Hs.367727; Mm.478333; Rn.50944 & *PGK2 & Phosphoglycerate kinase 2 & Human, mouse, rat \\
\hline Hs.129293; Mm.61148; Rn.59403 & *FAM71A & Family with sequence similarity 71 , member A Human, mouse, rat & \\
\hline Hs.534390; Mm.251434; Rn.63585 & ACTL7B & Actin-like 7B & Human, mouse, rat \\
\hline Hs. $189184 ;$ Mm.334092; Rn.66649 & UBQLN3 & Ubiquilin 3 & Human, mouse, rat \\
\hline Hs.629246; Mm.7411; Rn.81074 & HDGFL1 & Hepatoma derived growth factor-like 1 & Human, mouse, rat \\
\hline
\end{tabular}


Table 4. Continued

\begin{tabular}{|c|c|c|c|}
\hline Unigene & Gene name & Gene description & Species \\
\hline Hs.665663; Mm.121570 & MARCH10 & Membrane-associated ring finger 10 & Human, mouse \\
\hline Hs.2042; Mm.1296 & CRISP2 & Cysteine-rich secretory protein 2 & Human, mouse \\
\hline Hs.131654; Mm.141324 & DMRTB1 & DMRT-like family B with proline-rich C-terminal, 1 & Human, mouse \\
\hline Hs.97633; Mm.1498 & *AKAP4 & A kinase anchor protein 4 & Human, mouse \\
\hline Hs.209206; Mm.154333 & *ACTL9 & Actin-like 9 & Human, mouse \\
\hline Hs.303923; Mm.159193 & TKTL2 & Transketolase-like 2 & Human, mouse \\
\hline Hs.67639; Mm.159949 & *NUP210L & Nucleoporin $210 \mathrm{kDa}$-like & Human, mouse \\
\hline Hs.567774; Mm. 160714 & CCDC83 & Coiled-coil domain containing 83 & Human, mouse \\
\hline Hs.654377; Mm.16563 & *LDHC & Lactate dehydrogenase $\mathrm{C}$ & Human, mouse \\
\hline Hs.302028; Mm.173395 & DPEP3 & Dipeptidase 3 & Human, mouse \\
\hline Hs.298312; Mm.179050 & HORMAD1 & HORMA domain containing 1 & Human, mouse \\
\hline Hs.472491; Mm.189660 & OXCT2 & 3-Oxoacid CoA transferase 2 & Human, mouse \\
\hline Hs.122511; Mm.195831 & CETN1 & Centrin, EF-hand protein, 1 & Human, mouse \\
\hline Hs. $121021 ;$ Mm.196313 & SPATA3 & Spermatogenesis associated 3 & Human, mouse \\
\hline Hs.304020; Mm.196315 & CALR3 & Calreticulin 3 & Human, mouse \\
\hline Hs.663128; Mm.23515 & *ROPN1B & Ropporin, rhophilin associated protein 1B & Human, mouse \\
\hline Hs.414648; Mm.23534 & *TEKT3 & Tektin 3 & Human, mouse \\
\hline Hs.112743; Mm.243849 & SYCP1 & Synaptonemal complex protein 1 & Human, mouse \\
\hline Hs.405659; Mm.272720 & PIWIL1 & Piwi-like 1 & Human, mouse \\
\hline Hs.723454; Mm.279939 & *TXNDC3 & Thioredoxin domain containing 3 & Human, mouse \\
\hline Hs.169797; Mm.293080 & BOLL & Bol, boule-like & Human, mouse \\
\hline Hs.147816; Mm.30494 & MDH1B & Malate dehydrogenase $1 \mathrm{~B}, \mathrm{NAD}$ & Human, mouse \\
\hline Hs. $123530 ;$ Mm.30959 & *ACTL7A & Actin-like $7 \mathrm{~A}$ & Human, mouse \\
\hline Hs.444236; Mm.330943 & SPATA16 & Spermatogenesis associated 16 & Human, mouse \\
\hline Hs.367764; Mm.33646 & POM121L1P & POM121 membrane glycoprotein-like 1 pseudogene & Human, mouse \\
\hline Hs.2633; Mm.37953 & DSG1 & Desmoglein 1 & Human, mouse \\
\hline Hs.454835; Mm.440232 & TTLL10 & Tubulin tyrosine ligase-like family, member 10 & Human, mouse \\
\hline Hs.491885; Mm.45615 & DNAJC5B & DnaJ homolog, subfamily $\mathrm{C}$, member 5 beta & Human, mouse \\
\hline Hs. $151167 ;$ Mm.46125 & *PPP3R2 & Protein phosphatase 3 , regulatory subunit $\mathrm{B}$, beta isoform & Human, mouse \\
\hline Hs.121494; Mm.4688 & *SPAM1 & Sperm adhesion molecule 1 & Human, mouse \\
\hline Hs.210377; Mm.477086 & $\mathrm{CCDC} 38$ & Coiled-coil domain containing 38 & Human, mouse \\
\hline Hs. $169222 ;$ Mm.4776 & ACRV1 & Acrosomal vesicle protein 1 & Human, mouse \\
\hline Hs.97542; Mm.50808 & PLCZ1 & Phospholipase C, zeta 1 & Human, mouse \\
\hline Hs.412818; Mm.56514 & FAM71D & Family with sequence similarity 71 , member D & Human, mouse \\
\hline Hs.378893; Mm.61243 & TTC29 & Tetratricopeptide repeat domain 29 & Human, mouse \\
\hline Hs.388827; Mm.79070 & RFX4 & Regulatory factor X, 4 & Human, mouse \\
\hline Hs.398196; Mm.84725 & MYCBPAP & MYCBP associated protein & Human, mouse \\
\hline Hs.490715; Mm.87352 & RNF32 & Ring finger protein 32 & Human, mouse \\
\hline Hs. $126780 ; \mathrm{Mm} .87382$ & SEPT12 & Septin 12 & Human, mouse \\
\hline Hs.388841; Mm.9500 & $*$ ZPBP & Zona pellucida binding protein & Human, mouse \\
\hline Hs.320892; Mm.66952 & Adcy 10 & Adenylate cyclase 10 & Human, mouse \\
\hline Hs.411239; Rn.160028 & *KLKBL4 & Plasma kallikrein-like protein 4 & Human, rat \\
\hline Hs.307086; Rn.162868 & FSCB & Fibrous sheath CABYR binding protein & Human, rat \\
\hline Hs.350671; Rn.209597 & TSGA10IP & Testis-specific, 10 interacting protein & Human, rat \\
\hline Hs.644625; Rn.215025 & LRRIQ3 & Leucine-rich repeats and IQ motif containing 3 & Human, rat \\
\hline Hs.435371; Rn.33200 & *TCP11 & T-complex 11 homolog & Human, rat \\
\hline Hs. $177959 ;$ Rn.42917 & ADAM2 & ADAM metallopeptidase domain 2 & Human, rat \\
\hline Hs.98848; Rn.42918 & ADAM3A & ADAM metallopeptidase domain 3A & Human, rat \\
\hline Hs.367245; Rn.51329 & ZPBP2 & Zona pellucida binding protein 2 & Human, rat \\
\hline Hs. $240053 ;$ Rn. 68598 & GSG1 & Germ cell associated 1 & Human, rat \\
\hline Hs.655170; Rn.95004 & SPAG6 & Sperm associated antigen 6 & Human, rat \\
\hline Mm.143889; Rn.92321 & Adam5 & A disintegrin and metallopeptidase domain 5 & Mouse, rat \\
\hline Mm.17413; Rn.219514 & Fam $178 b$ & Family with sequence similarity 178, member B & Mouse, rat \\
\hline Mm.189668; Rn.122594 & Ubqlnl & Ubiquilin-like & Mouse, rat \\
\hline Mm.250465; Rn.26693 & Lrrc46 & Leucine rich repeat containing 46 & Mouse, rat \\
\hline Mm.255732; Rn.122669 & *Txndc2 & Thioredoxin domain containing 2 & Mouse, rat \\
\hline Mm.263615; Rn.123383 & *Spesp1 & Sperm equatorial segment protein 1 & Mouse, rat \\
\hline Mm.269223; Rn.170874 & Adam32 & A disintegrin and metallopeptidase domain 32 & Mouse, rat \\
\hline Mm.270295; Rn. 123187 & Tuba3b & Tubulin, alpha $3 \mathrm{~B}$ & Mouse, rat \\
\hline Mm.282330; Rn.27474 & *Tekt4 & Tektin 4 & Mouse, rat \\
\hline Mm.29788; Rn.47152 & Slfnl1 & Schlafen like 1 & Mouse, rat \\
\hline Mm.310201; Rn.163190 & Tssk2 & Testis-specific serine kinase 2 & Mouse, rat \\
\hline Mm.31708; Rn.3253 & Fscn3 & Fascin homolog 3 , actin-bundling protein, testicular & Mouse, rat \\
\hline Mm.432087; Rn.138402 & Tcp $10 b$ & T-complex protein $10 \mathrm{~b}$ & Mouse, rat \\
\hline Mm.436562; Rn.64496 & *Gapdhs & Glyceraldehyde-3-phosphate dehydrogenase, spermatogenic & Mouse, rat \\
\hline Mm.439725; Rn.114052 & Tssk1 & Testis-specific serine kinase 1 & Mouse, rat \\
\hline Mm.442295; Rn.172733 & Fbxl13 & F-box and leucine-rich repeat protein 13 & Mouse, rat \\
\hline Mm.52511; Rn.51791 & Atp8b3 & ATPase, class I, type $8 \mathrm{~B}$, member 3 & Mouse, rat \\
\hline Mm.61330; Rn.201753 & Gtf2a11 & General transcription factor IIA, 1-like & Mouse, rat \\
\hline Mm.67300; Rn.107052 & Ankrd36 & Ankyrin repeat domain 36 & Mouse, rat \\
\hline Mm.87067; Rn.27773 & *Spata18 & Spermatogenesis associated 18 & Mouse, rat \\
\hline Mm.87748; Rn.38091 & *Akap3 & A kinase anchor protein 3 & Mouse, rat \\
\hline
\end{tabular}

Total of 92 genes were overlapped in human, mouse and rat testis (see Fig. 1), asterisk (*) indicated common genes in the human sperm proteome (ref. 16). 
33 human testis-specific genes in this study were present in human sperm proteome which maybe play critical role in sperm biology. Integrated testis-specific genes from different species would provide supplementary information for insight into testicular physiology and functions.

RT-PCR analysis of thirteen randomly selected human genes showed that ten genes were exclusively expressed in the human testis, and three were weakly expressed in other organs which maybe have alternative splicing ${ }^{19)}$ or have too lower expression levels in other organs to be detected in transcriptome. Due to the limitation of tissue source, only 12 or-

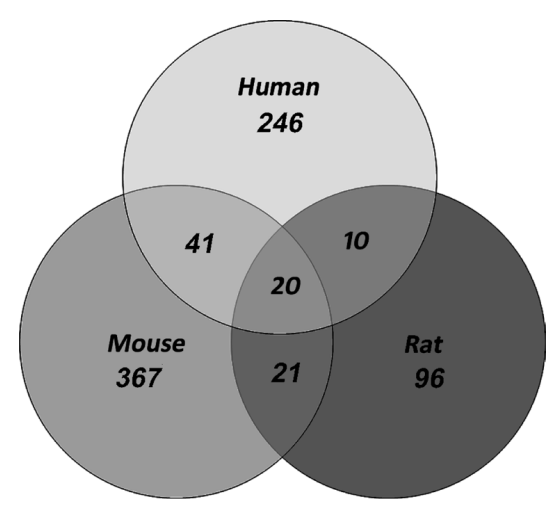

Fig. 1. Overlap of Testis-Specific Genes among Human, Mouse and Rat

The overlap of human (317), mouse (449) and rat (147) testis-specific genes are shown. The information of common genes in the three group are shown in Table 4. gans from 18 organs were validated, but these results could also prove the authenticity of the results. Interestingly, two homolog genes to human from mouse and rat were not screened in the human ESTs, but exclusively expressed in human testis by RT-PCR detection. The results indicated that some homolog genes to human from mouse and rat could provide complementary information for human testis studies and contribute to a more comprehensive human testis-specific gene profiles.

All testis-specific genes were performed gene-enrichment and functional annotation analysis through DAVID tools, a comprehensive analysis of the results showed that human, mouse and rat testis-specific genes have some common functional enrichments which were mainly related to spermatogenesis and development process with significant Glycolysis and Pyruvate metabolism. Testicular cells are different from other somatic cell which has special missions (spermatogenesis and steroidogenesis). Therefore, testis-specific genes of different species also have features in common.

Interestingly, differential enrichment analysis of protein domains through "PIR_SUPERFAMILY" revealed some significant superfamilies related to sperm function and fertilization potentially. Septin 12 and 14 were significantly expressed in testis which belongs to a family of polymerizing guanosine 5'-triphosphate (GTP)-binding proteins that are required for many cellular functions, such as membrane compartmentalaization, vesicular trafficking, mitosis and cytoskeletal remodeling, ${ }^{20)}$ for example, septin12 is critical for

Table 5. Thirty Three Genes Overlapped in Human Testis-Specific and Human Sperm Gene Database

\begin{tabular}{|c|c|c|c|}
\hline Unigene & Gene name & Gene description & Chromosome \\
\hline Hs. 121494 & SPAM1 & Sperm adhesion molecule 1 & 7 \\
\hline Hs. 123530 & ACTL7A & Actin-like 7A & 9 \\
\hline Hs. 126838 & ADAM29 & ADAM metallopeptidase domain 29 & 4 \\
\hline Hs. 129293 & FAM71A & Family with sequence similarity 71 , member A & 1 \\
\hline Hs. 143519 & TEKT5 & Tektin 5 & 16 \\
\hline Hs. 149030 & SLC25A31 & Solute carrier family 25 , member 31 & 4 \\
\hline Hs. 151167 & PPP3R2 & Protein phosphatase 3 , regulatory subunit $\mathrm{B}$, beta isoform & 9 \\
\hline Hs. 155942 & TRPM1 & Transient receptor potential cation channel, subfamily M, member 1 & 15 \\
\hline Hs. 179646 & TMCO5A & Transmembrane and coiled-coil domains 5A & 15 \\
\hline Hs.209206 & ACTL9 & Actin-like 9 & 19 \\
\hline Hs. 236635 & ACTRT2 & Actin-related protein $\mathrm{T} 2$ & 1 \\
\hline Hs. 307052 & LDHAL6B & Lactate dehydrogenase A-like 6B & 15 \\
\hline Hs. 348618 & TRIML1 & Tripartite motif family-like 1 & 4 \\
\hline Hs. 367727 & PGK2 & Phosphoglycerate kinase 2 & 6 \\
\hline Hs.388841 & ZPBP & Zona pellucida binding protein & 7 \\
\hline Hs.411239 & KLKBL4 & Plasma kallikrein-like protein 4 & 16 \\
\hline Hs. 414648 & TEKT3 & Tektin 3 & 17 \\
\hline Hs. 435371 & TCP11 & T-complex 11 homolog & 6 \\
\hline Hs. 511983 & CABYR & Calcium binding tyrosine-phosphorylation regulated & 18 \\
\hline Hs.522093 & C9orf144B & Hypothetical protein LOC 259308 & 9 \\
\hline Hs.525769 & C15orf55 & Chromosome 15 open reading frame 55 & 15 \\
\hline Hs.591011 & FAM71C & Family with sequence similarity 71 , member $\mathrm{C}$ & 12 \\
\hline Hs. 643584 & IGSF10 & Immunoglobulin superfamily, member 10 & 3 \\
\hline Hs. 654377 & LDHC & Lactate dehydrogenase $\mathrm{C}$ & 11 \\
\hline Hs.662219 & ATP1A4 & ATPase, $\mathrm{Na}+/ \mathrm{K}+$ transporting, alpha 4 polypeptide & 1 \\
\hline Hs. 663128 & ROPN1B & Ropporin, rhophilin associated protein 1B & 3 \\
\hline Hs.666099 & FAM71B & Family with sequence similarity 71 , member B & 5 \\
\hline Hs.67639 & NUP210L & Nucleoporin $210 \mathrm{kDa}$-like & 1 \\
\hline Hs. 701555 & TSSK1B & Testis-specific serine kinase $1 \mathrm{~B}$ & 5 \\
\hline Hs.723454 & TXNDC3 & Thioredoxin domain containing 3 & 7 \\
\hline Hs.97633 & AKAP4 & A kinase anchor protein 4 & $\mathrm{X}$ \\
\hline Hs. 98008 & GK2 & Glycerol kinase 2 & 4 \\
\hline Hs. 98712 & TXNDC2 & Thioredoxin domain containing 2 & 18 \\
\hline
\end{tabular}



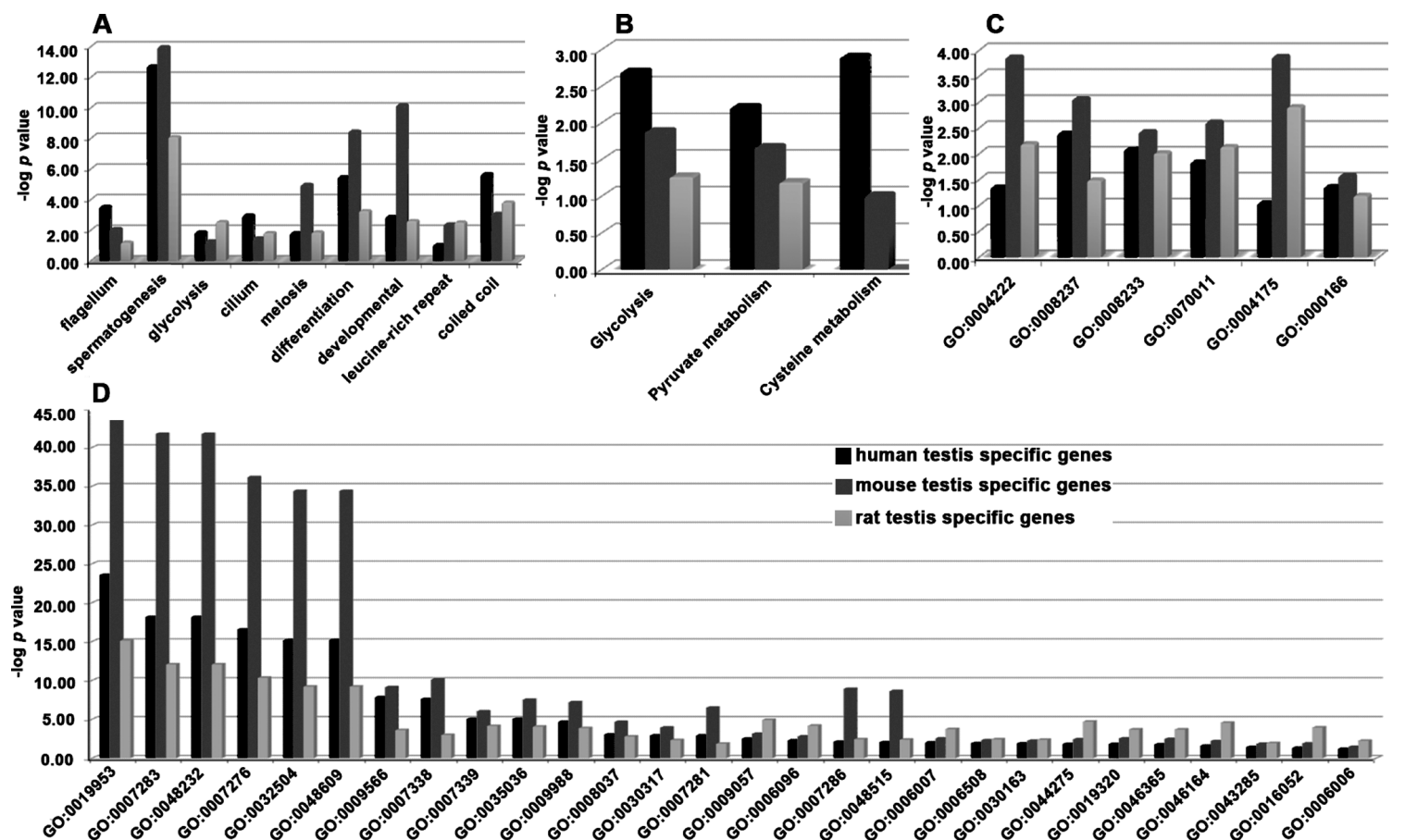

Fig. 2. Common Enrichment Differential Functions among Human, Mouse and Rat Testis-Specific Genes Analysis through DAVID

(A) PIR keywords; (B) KEGG pathways; (C) molecular functions; (D) biology processes. Vertical coordinates represent the $p$ values. Horizontal ordinate represent statistically significant functions.

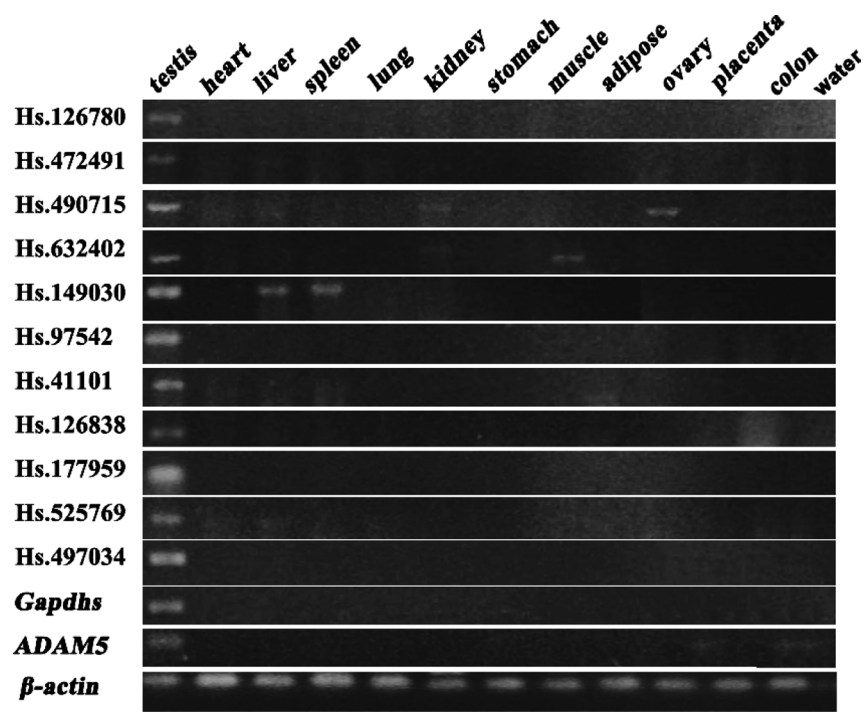

Fig. 3. RT-PCR Results of Thirteen Selected Testis-Specific Genes in Different Human Organs

mRNA extraction from 12 human organs were used to identify testis-specific genes expression; $\beta$-actin was the positive control; water instead of DNA was the negative control.

spermiogenesis. $^{21)}$ Tektin family had been verified to be present in sperm flagella which may be involved in flagellar stability and sperm motility. ${ }^{22)}$ In this study, four tektin genes were found including Tekt2 (Rat), Tekt5 (human), Tekt3 (human and mouse) and tekt4 (mouse and rat). A Disintegrin and Metalloprotease (ADAM) family members were expressed in male reproductive tissues which were involved in fertilization process. $^{23)}$ Some ADAMs in human (3 genes), mouse ( 9 genes) and rat (5 gene) were detected specifically in the testis, three ADAM genes were validated by RT-PCR.
These ADAMs genes maybe play important role in the sperm maturation process.

In conclusion, we present a systematic mapping of testisspecific genes from human, mouse and rat unigene libraries, the combination of testis-specific genes should serve as a source of reference for future research and clinical application. Further studies are warranted to substantiate the expression of mouse and rat homologue genes in human testis. Such studies will advance our understanding of testis physiology and function, also facilitate studies of sperm maturation.

\section{REFERENCES}

1) Swerdloff R. S., Wang C., Bhasin S., Baillieres Clin. Endocrinol. Metab., 6, 451-483 (1992).

2) Dufau M. L., Tsai-Morris C., Tang P., Khanum A., J. Steroid Biochem. Mol. Biol., 76, 187-197 (2001).

3) Walker W. H., Steroids, 74, 602-607 (2009).

4) Bettoni F., Filho F. C., Grosso D. M., Galante P. A., Parmigiani R. B., Geraldo M. V., Henrique-Silva F., Oba-Shinjo S. M., Marie S. K., Soares F. A., Brentani H. P., Simpson A. J., de Souza S. J., Camargo A. A., Genomics, 94, 153-160 (2009).

5) Liu L., Smith J. W., Endocrinology, 141, 2033-2042 (2000).

6) Pinheiro A., Faustino I., Silva M. J., Silva J., Sá R., Sousa M., Barros A., de Almeida I.T., Rivera I., Mol. Genet. Metab., 99, 425-430 (2010).

7) Ghafouri-Fard S., Abbasi A., Moslehi H., Faramarzi N., Taba Taba Vakili S., Mobasheri M. B., Modarressi M. H., Br. J. Dermatol., 162, $772-779$ (2009).

8) Hillmann A., Dunne E., Kenny D., Methods Mol. Biol., 496, 223-243 (2009).

9) Fook L. W., Chow V. T., Methods Mol. Biol., 630, 33-47 (2010).

10) Le K., Mitsouras K., Roy M., Wang Q., Xu Q., Nelson S. F., Lee C., Nucleic Acids Res., 32, e180 (2004).

11) von Kopylow K., Kirchhoff C., Jezek D., Schulze W., Feig C., Primig M., Steinkraus V., Spiess A. N., Hum. Reprod., 25, 1104-1112 (2010). 
12) Scheurle D., DeYoung M. P., Binninger D. M., Page H., Jahanzeb M., Narayanan R., Cancer Res., 60, $4037-4043$ (2000).

13) Wang N., Xue L., Yuan A., Xu D., Exp. Anim., 58, 547—556 (2009).

14) O’Bryan M. K., de Kretser D., Int. J. Androl., 29, 76-89 (2006).

15) Li J. Y., Wang H. Y., Liu J., Liu Q., Zhang J. S., Wan F. C., Liu F. J., Jin S. H., Zhang Y. L., DNA Res., 15, 115-122 (2008).

16) Baker M. A., Reeves G., Hetherington L., Müller J., Baur I., Aitken R. J., Proteom. Clin. Appl., 1, 524-532 (2007).

17) Kim N., Lee C., Methods Mol. Biol., 452, 179-197 (2008).

18) Tang A., Yu Z., Gui Y., Zhu H., Zhang L., Zhang J., Cai Z., Biol. Pharm. Bull., 30, 865-872 (2007).
19) Hallegger M., Llorian M., Smith C. W., FEBS J., 277, 856-866 (2010).

20) Cao L., Yu W., Wu Y., Yu L., Cell. Mol. Life Sci., 66, 3309-3323 (2009).

21) Lin Y. H., Lin Y. M., Wang Y. Y., Yu I. S., Lin Y. W., Wang Y. H., Wu C. M., Pan H. A., Chao S. C., Yen P. H., Lin S. W., Kuo P. L., Am. J. Pathol., 174, 1857-1868 (2009).

22) Amos L. A., Genome Biol., 9, 229 (2008).

23) Han C., Choi E., Park I., Lee B., Jin S., Kim do H., Nishimura H., Cho C., Biol. Reprod., 80, 1001-1008 (2009). 